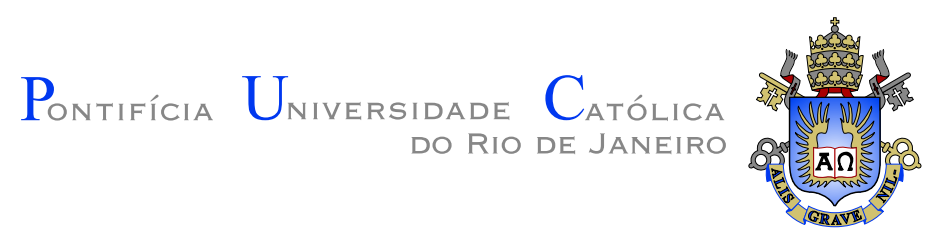

Nei Cipriano Ribeiro

\title{
Estudo dos Efeitos da Interação Não Padrão dos Neutrinos com a Matéria em Experimentos de Oscilaçãos de Neutrinos de Grandes Comprimentos
}

\section{Tese de Doutorado}

Tese apresentada ao Programa de Pós-graduação em Física do Departamento de Física da PUC-Rio como requisito parcial para obtenção Do título de Doutor em Física

Orientador: Prof. Hiroshi Nunokawa 


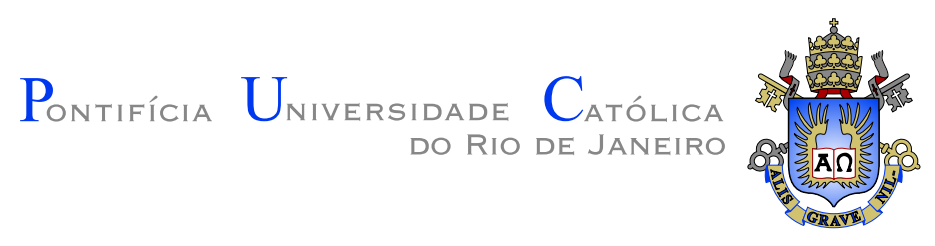

Nei Cipriano Ribeiro

\begin{abstract}
Estudo dos Efeitos da Interação Não Padrão dos Neutrinos com a Matéria em Experimentos de Oscilaçãos de Neutrinos de Grandes

Comprimentos
\end{abstract}

Tese apresentada ao Programa de Pós-graduação em Física do Departamento de Física do Centro Técnico Científico da PUCRio como requisito parcial para obtenção Do título de Doutor em Física. Aprovada pela Comissão Examinadora abaixo assinada.

\author{
Prof. Hiroshi Nunokawa \\ Orientador \\ Departamento de Física - PUC-Rio
}

Prof. Carla Göbel Burlamaqui de Mello Departamento de Física - PUC-rio

Prof. João Carlos Costa dos Anjos CBPF

Prof. Oscar josé Pinto Éboli

USP

Prof. Orlando Luis Goulart Peres

UNICAMP

Prof. José Eugênio Leal

Coordenador Setorial do Centro Técnico Científico - PUC-Rio 
Todos os direitos reservados. É proibida a reprodução total ou parcial do trabalho sem autorização da universidade, do autor e do orientador.

\section{Nei Cipriano Ribeiro}

Graduou-se em Bacharelado e em Licenciatura plena em Física na Universidade Estadual do Rio de Janeiro em 2001. Mestrou-se em Física pela Universidade Estadual do Rio de Janeiro em 2003.

Ficha Catalográfica

Cipriano Ribeiro, Nei

Estudo dos Efeitos da Interação Não Padrão dos Neutrinos com a Matéria em Experimentos de Oscilaçãos de Neutrinos de Grandes Comprimentos / Nei Cipriano Ribeiro; orientador: Hiroshi Nunokawa. - Rio de Janeiro : PUC-Rio, Departamento de Física, 2008.

v., 136 f: il. ; $29,7 \mathrm{~cm}$

1. Tese (doutorado) - Pontifícia Universidade Católica do Rio de Janeiro, Departamento de Física.

Inclui referências bibliográficas.

1. Física - Tese. 2. Oscilação de Neutrinos. 3. Física além do Modelo Padrão. 4. Interação não padrão. 5. Violação de CP. 6. Fábrica de Neutrinos.

I. Nunokawa, Hiroshi. II. Pontifícia Universidade Católica do Rio de Janeiro. Departamento de Física. III. Título. 


\section{Agradecimentos}

A Deus.

Ao meu orientador e amigo Prof. Dr Hiroshi Nunokawa.

Ao $\mathrm{CNPq}$ e à PUC-Rio, pelos auxílios concedidos, sem os quais este trabalho não poderia ter sido realizado.

À minha família, em particular minha mãe, Clarice; minha esposa, Andreza, e minhas filhas: Rabech e Raíssa, o qual são fontes eternas de inspiração.

Aos meus colegas da PUC-Rio, quem me fizeram adorar esse lugar.

Aos professores Renata Zukanovich Funchal (USP) e Hisakazu Minakata (Univ. Metropolitana de Tóquio) pelos conhecimentos transmitidos e por suas valiosas ajudas, que foram fundamentais para realização desta obra.

Ao CEFETCAMPOS, em particular ao Prof. Dr Paulo Colato e as coordenadoras de Ensinos: do Superior, Rita Daher e do Ensino Médio Marília pela compreensão e incentivo.

Ao pessoal do departamento de Física pela ajuda de todos os dias, em particular às secretárias Giza, Márcia e Majô; e também ao amigo e irmão julinho pelo carinho e presteza de sempre. 


\section{Resumo}

Cipriano Ribeiro, Nei; Nunokawa, Hiroshi. Estudo dos Efeitos da Interação Não Padrão dos Neutrinos com a Matéria em Experimentos de Oscilaçãos de Neutrinos de Grandes Comprimentos. Rio de Janeiro, 2008. 136p. Tese de Doutorado - Departamento de Física, Pontifícia Universidade Católica do Rio de Janeiro.

Em física de neutrinos, quase todos os dados de experimentos importantes podem ser explicados através de oscilação de neutrinos causados por massas e misturas. Porém, existe a possibilidade de que os neutrinos tenham algumas propriedades, além dessas mencionadas, que manifesta alguma física nova ou física além do modelo padrão. Esta nova física pode ser explicada por partículas exóticas ainda desconhecidas que são responsáveis por interações extras ou interação não padrão com os neutrinos e aqui as estudaremos fenomenologicamente, isto é, não nos preocuparemos com um modelo teórico em si. Nesta tese, estudamos os efeitos dessa interação não-padrão de neutrinos com a matéria para os experimentos de oscilação de grandes comprimentos. Nós consideramos dois detetores idênticos, mas com suas distâncias em relação à fonte, diferentes em dois casos: Primeiro, estudamos o potencial para sondar os efeitos de interação não-padrão para experimentos que usam feixes de neutrinos convencionais vindo de decaimentos de píons. Como exemplo, consideramos um feixe de neutrinos a ser criado pelo acelerador de prótons do JPARC (Japan Proton Accelerator Research Complex) no Japão. Nele, consideramos dois detetores, além do detetor próximo ao JPARC para monitorar o feixe; temos o primeiro que está situado em Kamioka, Japão e o outro em algum lugar na Coréia do Sul, sendo que o primeiro está a $295 \mathrm{~km}$, enquanto o segundo está em torno de $1000 \mathrm{~km}$ do JPARC. Segundo, avaliamos também o potencial da fábrica de neutrinos, o qual utiliza feixes de neutrinos vindo do decaimento de múons armazenados num anel para estudar os parâmetros de interação não-padrão e o seu impacto na sensibilidade da fase de violação CP e o ângulo de mistura $\theta_{13}$. Neste trabalho, também combinamos os dados simulados com dois detetores com comprimentos diferentes, sendo o primeiro a $3000 \mathrm{~km}$ e o outro a $7000 \mathrm{~km}$ da fonte. O comprimento deste segundo detetor foi escolhido pelo fato de ter uma característica peculiar, o Magic Baseline (comprimento mágico), onde a probabilidade de oscilação não depende da fase de violação de CP quando os parâmetros não-padrão estão ausentes.

\section{Palavras-chave}

Oscilação de Neutrinos. Física além do Modelo Padrão. Interação não padrão. Violação de CP. Fábrica de Neutrinos. 


\section{Abstract}

Cipriano Ribeiro, Nei; Nunokawa, Hiroshi. Probing of Non Standard Neutrinos Interation with Matter in Oscilation Neutrino Experiments with Long Baseline. Rio de Janeiro, 2008. 136p. PhD Thesis - Department of Física, Pontifícia Universidade Católica do Rio de Janeiro.

In neutrino physics, most of the experimental data importants can be explained due to neutrino oscilation induced by mass and mixing. However, there is the posibility that the neutrinos have some properties, new physics or beyond Standard Model. This new physics can be explained with some so far unknown exotic particles that are responsables for extra interactions or non standard interaction with neutrinos and here we studied fenomenologically, without concerning about any theoretical model. In this Thesis, we study the effects of Non Standard Interaction (NSI) with matter in experiments of oscilation in long baseline. We consider two identical detectors, but wih diferent baselines from source, in two cases: first, we study the probing potencial to analyse the NSI effect for experiments that use conventional neutrino beams coming from pions decay. For example, we should consider a neutrino beam created at the Proton Accelerator JPARC (Japan Proton Accelerator Research Complex) in Japan. We consider two detectors, in addition to the front detector at JPARC to monitor the beam; we have the first in Kamioka, Japan and other somewhere in Korea, the first is at 295 $\mathrm{km}$, and the second is localizate about $1000 \mathrm{~km}$ from JPARC. In sequence, we also evaluate the potencial of Neutrino Factory, where a neutrino beam come from a muon decay storage ring, in order to study the non standard interactions parameter and its impact in CP phase sensibility and the mixing angle $\theta_{13}$. In this work we combined theses simulated data from two detectors with differents baselines, with the first localized at $3000 \mathrm{~km}$ and the other at $7000 \mathrm{~km}$ from the source. The baseline of this second detector was chosen because it has a special feature, the Magic Baseline, where the oscilation probability do not depend of $\mathrm{CP}$ phase violation when the non standard parameters are absent.

\section{Keywords}

Neutrino Oscilation. Non-Standard Interaction. CP Violation. Neutrinos Factory. 


\section{Sumário}

1 Introdução 16

1.1 Aspectos históricos da física de Neutrinos 16

1.2 Motivação e Objetivo 18

2 Conhecimentos Básicos de Neutrinos $\quad 21$

2.1 Neutrinos no Modelo Padrão 21

2.2 Natureza de Massas dos Neutrinos 23

3 O Mecanismo de Oscilação dos Neutrinos $\quad 27$

3.1 Oscilação em 2 Gerações 28

3.2 Oscilação em 3 Gerações 33

4 As fontes de neutrinos e os Principais Resultados de Oscilação $\quad 38$

4.1 Neutrinos solares 38

4.2 Neutrinos atmosféricos 43

4.3 Neutrinos de reatores $\quad 45$

4.4 Neutrinos de aceleradores $\quad 50$

5 O Mecanismo de Oscilação com os Termos de Interação Não Padrão 56

5.1 Introdução 56

5.2 Limites Experimentais para os parâmetros NSI 58

6 Experimentos de Futuros Aceleradores de Grandes

Comprimentos $\quad 64$

6.1 T2K 64

6.2 NOvA 66

6.3 Fábricas de Neutrinos 67

7 Sondagem do NSI usando feixes de neutrinos convencionais:

$\begin{array}{ll}\text { T2KK } & 71\end{array}$

$\begin{array}{lll}7.1 & \text { Introdução } & 71\end{array}$

7.2 Método de análise $\quad 72$

7.3 O Efeito da Interação Não Padrão 73

$\begin{array}{lll}7.4 & \text { Resultados } & 75\end{array}$

8 Sondagem do NSI usando feixes de neutrinos não convencionais: Fábrica $\begin{array}{ll}\text { de Neutrinos } & \mathbf{8 0}\end{array}$

8.1 Introdução $\quad 80$

8.2 O Comprimento Mágico (Magic Baseline) 80

8.3 Os efeitos de NSI no Comprimento Mágico 82

8.4 Estudo do efeito NSI no detetor a $\mathrm{L}=3000 \mathrm{~km}$

8.5 Detetor a $L=3000 \mathrm{~km}$ e a sinergia esperada quando combinada com outro detetor a $L=7000 \mathrm{~km}$

8.6 Método de análise 86

8.7 Tratamento Estatístico $\quad 87$ 
8.8 Sensibilidades dos Parâmetros NSI 88

8.9 Determinação dos parâmetros NSI 104

8.10 Parâmetros de degenerescência com e sem NSI 104

$\begin{array}{ll}8.11 \text { Análise dos alcances para NSI } & 106\end{array}$

9 Conclusão e Trabalhos Futuros $\quad \mathbf{1 1 5}$

$\begin{array}{lll}\text { A Obtenção do Potencial Efetivo } & 127\end{array}$

B Obtenção da Equação de Movimento na Matéria em 2
gerações

C O obtenção da Probabilidade de Oscilação Aproximado com Efeito Não Padrão pelo método KTY 


\section{Lista de figuras}

2.1 Diagramas de espalhamento do neutrino através de interações de correntes carregadas CC e neutra NC.

3.1 As massas efetivas adquiridas por um sistema com 2 neutrinos em função do potencial efetivo $a$ (conforme a Eq. 3-20). Adaptado da Ref. (69).

3.2 O ângulo de mistura na matéria para um sistema de dois neutrinos em função do potencial $A$ para 2 diferentes ângulos de misturas no vácuo [conforme a Eq. (3-21)], Ref. (69).

3.3 Os ordenamentos de massas dos neutrinos, sendo que na figura da esquerda temos a hierarquia normal enquanto a da direita, a hierarquia invertida.

4.1 As distribuições de energia dos fluxos de neutrinos solar previsto pelo SSM (5). Os alcance das energias associados com os vários experimentos são indicados no topo da figura.

4.2 Os fluxos de neutrinos solares observados pela media de corrente carregada, corrente neutra e espalhamento elastico no SNO e pela medida do espalhamento no SK. Adaptado da Ref. (27).

4.3 As regiões permitidas dos parametros de oscilação baseado em dados de apenas SNO (figura esquerda), de todos os experimentos de neutrinos solares (figura direita superior) e de todos os experimentos de neutrinos solares mais de KamLAND (figura direita inferior). Adaptada da Ref. (27).

4.4 Distribuições dos eventos em função do ângulo zenital. Os pontos são os dados, os histogramas são os eventos simulados por Monte Carlo (MC) e as linhas representam os melhores ajustes esperados para oscilações $\nu_{\mu} \leftrightarrow \nu_{\tau}$ com os seguintes parâmetros $\sin ^{2} 2 \theta=1.0$ e $\Delta m^{2}=2.1 \times 10^{-3} \mathrm{eV}^{2}$. A largura dos retângulos mostra o erro estatístico do MC. Adaptado da Ref. (80).

4.5 Número de eventos normalizados dos neutrinos atmosférico como função de distância percorrida pelo neutrino/energia. Adaptado da Ref. (19).

4.6 Regiões permitidas dos parâmetros de oscilações a 68, 90 and 99 \% CL obtidas pela análise dos dados mostrados nas Fig. 4.4 e 4.5 . Adaptado da Ref. (80).

4.7 As regiões excluídas a $90 \%$ para oscilações $\nu_{e}$ dos experimentos de reatores e a sensibilidade esperadas do experimento KamLAND. Adaptada da Ref (99).

4.8 Números de eventos como função de energia do pósitron observados no detetor de KamLAND. Adaptada da Ref.(28). 
4.9 O lado esquerdo: as regiões permitidas dos dados de anti-neutrinos do kamLAND e de experimentos de neutrinos solares (linhas pontilhadas) para 1, 2,e $3 \sigma$. Lado direito: resultados combinados da análise de oscliações de 2 neutrinos do kamLAND e de neutrinos solares, admitindo invariância CPT. O ponto de melhor ajuste nos fornece $\Delta m^{2}=7.9_{-0,5}^{+0,6} \mathrm{eV}^{2} \times 10^{-5}$ e $\tan \theta=0.040_{-0,07}^{+0,10}$. Adaptada da Ref.(28).

4.10 As regiões permitidas dos parametros de oscilação para $68 \%, 90 \%$ e 99\% CL. Adaptado da Ref. (20).

4.11 Superior: A distribuição do número de eventos em função da energia (pontos), comparando com os dados simulados MC (linhas pontilhadas) e os dados sem oscilação. Inferior: A taxa $R$ entre os dados e os valores previstos em função da energia. Adaptado da Ref. (94).

4.12 As regiões permitidas a $68 \%, 90 \%$ e $99 \% \mathrm{CL}$ e a estrela mostra o ponto de melhor ajuste. Adotado pela Ref. (94).

5.1 O espaço de parâmetro $\varepsilon-\varepsilon^{\prime}$ para o mecanismo de oscilação com o efeito NSI. Os parâmetros $\sin ^{2} 2 \theta_{23}$ e $\Delta m_{32}^{2}$ foram marginalizados. As áreas correspondem a $90 \%, 95 \%$ e $99 \%$ e $99.73 \%$ CL respectivamente. $\mathrm{O}$ melhor ponto de ajuste é identificado pela estrela e os dados são dos MACRO+SK. Adaptado da Ref. (46).

5.2 As regiões permitidas para dois parâmetros livres e o parâmetro fixo $\varepsilon_{e e}$ para cada painel. Os ajustes foram obtidos combinando os dados do $\mathrm{K} 2 \mathrm{~K}$ e atmosféricos. Os valores dos parâmetros de oscilação $\Delta m_{32}^{2}$ e $\theta_{23}$ foram marginalizados. Também é usado $\theta_{23}=0$ e negligenciado $\Delta m_{21}^{2}$. Os contornos correspondem de dentro para fora a $95 \%, 99 \%$ e $3 \sigma$ respectivamentes. Adaptada da Ref. (100).

6.1 O layout do J-PARC que é composto por 3 aceleradores: o LINAC, $3 \mathrm{GeV}$ PS e o $50 \mathrm{GeV}$ PS. Adaptado da Ref. (101).

6.2 Esquema da fábrica de neutrinos. Adaptado da Ref.(107).

7.1 As probabilidades de oscilações padrão em vermelho e quando os parâmetros NSI estão ligados de acordo com a legenda. Na primeira coluna está Kamioka e na segunda Coréia, na primeira linha temos neutrinos e enquanto que na segunda linha temos antineutrinos. Observamos ao comparar os gráficos que que 0 impacto do parâmetro $\varepsilon_{\mu \tau}$ é muito maior na probabilidade de oscilação do que o parâmetro $\varepsilon_{\mu \tau}$.

7.2 As regiões permitidas no sistema $\varepsilon_{\mu \tau}-\varepsilon_{\tau \tau}$ para 4 anos de exposição, para neutrinos (painéis superiores), antineutrinos (painéis do meio) e a combinação de neutrino + antineutrinos (painéis inferiores). Em todos os casos são aplicados para Kamioka, Coréia e Kamioka+Coréia. Estamos tomando como dados de entrada $\varepsilon_{\mu \tau}$ e $\varepsilon_{\tau \tau}$ nulos e, considerando os parâmetros $\Delta m_{32}^{2}$ e $\theta_{23}$ fixos, iguais a $\Delta m_{32}^{2}=2.5 \times 10^{-3} \mathrm{eV}^{2}$ e $\sin ^{2} \theta_{23}=0.5$. A massa fiducial sendo a mesma em todos os casos igual a 0.54 Mtoneladas.

7.3 O mesmo da fig. $7.2 \operatorname{com} \theta_{23}$ variando. 
7.4 Estamos considerando os mesmos parâmetros da figura $7.2 \mathrm{com}$ $\theta_{23}$ e $\Delta m_{32}^{2}$ variando.

7.5 As regiões permitidas no sistema $\varepsilon_{\mu \tau}-\varepsilon_{\tau \tau}$ análogo a fig. 7.4, mas com massa fiducial de 0.54Mton para Kamioka e Coréia e 0.27 Mton quando combinado Kamioka +Coréia. Na primeira coluna corresponde $\sin ^{2} \theta_{23}=0.45$, enquanto na segunda $\sin ^{2} \theta_{23}=0.55$. Em todos os painéis já estão combinados neutrinos+antineutrinos. Na parte de Kamioka, tem melhor sensibilidade do que nas partes de Coréia e combinada, onde vemos múltiplas soluções de $\varepsilon_{\tau \tau}$ para $\sin ^{2} \theta_{23}=0.45$. As linhas vermelha, amarela e azul correspondem a $1 \sigma, 2 \sigma$ e $3 \sigma$, respectivamente.

8.1 O plano de bi-probabilidade $(P \times \bar{P})$ onde vemos que no $\mathrm{MB}$, $L \approx 7.200 \mathrm{Km}$, não há elipses como nos demais comprimentos. Portanto no MB não há dependência da fase CP na ausência do NSI. 81

8.2 O alcance da probabilidade de oscilação em função do comprimento do detetor, com a fase de violação CP variando de $0-2 \pi$.

8.3 O espaço de bi-probabilidades os impactos dos parâmetros NSI, ligados um por vez no MB com 3 valores de $\sin ^{2} 2 \theta_{13}=$ $0.0005,0.001$ e 0.0015 , rescpectivamente.A figura mostra que no MB pode-se resolver a degenerescência de $\theta_{13}$ com a fase de violação CP $\delta$.

8.4 O espaço de bi-probabilidades os impactos dos parâmetros NSI, ligados um por vez no MB com 3 valores de $\sin ^{2} 2 \theta_{13}=0.0005$, 0.001 e 0.0015 , rescpectivamente. Os mesmos parâmetros da Fig. 8.3, mais com $L=3000 \mathrm{~km}$ e densidade de matéria $\rho=3.6 \mathrm{~g} / \mathrm{cm}^{3}$ para $\varepsilon$ positivo e negativo.

8.5 As regiões permitidas dentro do plano de dois parâmetros NSI, $\varepsilon_{e e}-\varepsilon_{e \mu}$ (paineis esquerdos), $\varepsilon_{\tau \tau}-\varepsilon_{e \tau}$ (painel do centro) e $\varepsilon_{e e}-\varepsilon_{\tau \tau}$ (painel da direita) que corresponde o caso onde os dados de entrada são $\sin ^{2} 2 \theta_{13}=0.001$ e $\delta=\pi / 4$ e os parâmetros NSI nulos. Estamos considerando $E_{\mu}=50 \mathrm{GeV}$ e os comprimentos $\mathrm{L}=3000 \mathrm{~km}$ (painéis superiores), $\mathrm{L}=7000 \mathrm{~km}$ (painéis do centro) e combinação (painéis inferiores). As linhas pontinhadas representam os valores de de $\varepsilon_{\alpha \beta}$ nulo. O ajuste foi conseguido variando 4 parâmetros $\theta_{13}, \delta$ e 2 $\epsilon$ 's com $\theta_{13}, \delta$ maginalizados, isto é, minimizados. O número de decaimento de múons por anos é de $10^{21}$, o tempo de exposição é de 4 anos para neutrino e antineutrino, e cada detetor tem massa de 50kt. Estamos considerando apenas 3 bins. Os demais parâmetros de oscilações são fixados como: $\Delta m_{23}^{2}=2.5 \times 10^{-3} \mathrm{eV}^{2}$, $\sin ^{2} \theta_{23}=0.5$ e $\Delta m_{12}^{2}=8.0 \times 10^{-5}$ e $\sin ^{2} \theta_{12}=0.31$.

8.6 O mesmo da Fig. 8.5, mais com diferentes combinações dos $\varepsilon$ 's, $\varepsilon_{e e}-\varepsilon_{e \mu}$ (painel esquerdo), $\varepsilon_{\tau \tau}-\varepsilon_{e \mu}$ (painel do centro) e $\varepsilon_{e \mu}-\varepsilon_{e \tau}$ (painel direito).

8.7 O mesmo da 8.5 mas $\operatorname{com} \delta=3 \pi / 2$. 92

8.8 O mesmo da 8.6 mas com $\delta=3 \pi / 2$. 
8.9 As regiões permitidas projetada no plano de $\sin ^{2} 2 \theta_{13}-\delta$ correspondendo o caso onde o parâmetro de entrada são $\sin ^{2} \theta_{23}=0.001$ e $\delta=\pi / 4$ e os parâmetros NSI nulos. Para $E_{\mu}=50 \mathrm{GeV}$ e o comprimento $L=3000 \mathrm{~km}$ (painéis superiores), $L=7000 \mathrm{~km}$ (painéis do centro) e combinação (painéis inferiores). O ajuste foi calculado variando livremente $\theta_{13}, \delta$ e NSI, onde esses parâmetros foram marginalizados, $\varepsilon_{e e}-\varepsilon_{e \tau}$ (painel esquerdo), $\varepsilon_{\tau \tau}-\varepsilon_{e \tau}$ (painéis do meio) e $\varepsilon_{e e}-\varepsilon_{\tau \tau}$ (painéis da direita). $\mathrm{O}$ mesmo parâmetro de entrada e ajuste da Fig. 8.5

8.10 A mesma Fig. 8.9 mais para diferentes combinações de dois $\varepsilon$ 's. Temos as combinações $\varepsilon_{e e}-\varepsilon_{e \mu}$ (painéis da esquerda), $\varepsilon_{\tau \tau}-\varepsilon_{e \mu}$ (painéis do meio) e $\varepsilon_{e \mu}-\varepsilon_{e \tau}$ (painéis da esquerda).

$8.11 \mathrm{O}$ mesmo da Fig. 8.9 mas com $\delta=3 \pi / 2$.

8.12 O mesmo da Fig.8.10 mas com $\delta=3 \pi / 2$.

8.130 mesmo da Fig. 8.7 mas com $\sin ^{2} 2 \theta_{13}=0.0001$. 100

8.140 mesmo da Fig. 8.8 mas com $\sin ^{2} 2 \theta_{13}=0,0001$. 101

8.150 mesmo da Fig.8.11 mas com $\sin ^{2} 2 \theta_{13}=0.0001$. 102

8.16 O mesmo da Fig. 8.12 mas com $\sin ^{2} 2 \theta_{13}=0.0001$

8.17 As regiões permitidas projetada no plano de $\sin ^{2} 2 \theta_{13}-\delta$ para - caso de oscilação padrão sem o NSI, para valores de entrada $\sin ^{2} \theta_{13}=0.001$ e $\delta=\pi / 4$ (painéis da esquerda) e $\delta=3 \pi / 4$ (painéis da direita). A região permitida foi calculada com 2 DOF. A condição experimental é a mesma da Fig. 8.9.

$8.18 \mathrm{O}$ mesmo da Fig.8.7 mas com valores de entrada de NSI $\varepsilon, \varepsilon_{e e}=$ $0.1, \varepsilon_{e \tau}=0.01$ e $\varepsilon_{\tau \tau}=0.2$. A linhas pontilhadas correspondem valores não nulos de $\varepsilon_{\alpha \beta}$

8.190 mesmo da Fig.8.11, mais com dados de entrada $\varepsilon, \varepsilon_{e e}=$ $0.1, \varepsilon_{e \tau}=0.01$ e $\varepsilon_{\tau \tau}=0.2$.

8.20 Os isocontornos de $2 \sigma \mathrm{CL}$ (para $2 \mathrm{DOF}$ ) a sensibilidade para o parâmetro $\varepsilon_{\alpha \beta}$ em função dos valores de entrada dos parâmetros $\theta_{13}$ e $\delta$. Para cada ponto no plano $\theta_{13}-\delta$ a incerteza é definida por $\Delta \varepsilon \equiv\left(\varepsilon_{\max }-\varepsilon_{\min }\right) / 2$, onde $\varepsilon_{\max }$ ou $\varepsilon_{\min }$ indicam os valores máximo e mínimo para os valores de $\varepsilon$ o qual é consistente com caso sem efeito NSI. Nos paineis superior, do meio e inferior, as sensibilidades para (a1) $\varepsilon_{e e}$ e (a2) $\varepsilon_{e \mu}$ para o sistema $\left.\varepsilon_{e e}-\varepsilon_{e \mu}, \mathrm{b} 1\right)$ $\varepsilon_{e e}$ e (b2) $\varepsilon_{e \tau}$ para o sistema $\varepsilon_{e e}-\varepsilon_{e \tau}$, e (c1) $\varepsilon_{e e}$ e (c2) $\varepsilon_{\tau \tau}$ para o sistema $\varepsilon_{e e}-\varepsilon_{\tau \tau}$. Notamos que as incertezas para (a2) é $\times 100$, enquanto (b2) é $\times 10$.

8.21 O mesmo da Fig. 8.20 mas com diferença na combinaçã dos $\varepsilon^{\prime}$. Nos paineis superior, do centro e inferior, mostram as sensibilidades para (d1) $\varepsilon_{e \mu}$ e (d2) $\varepsilon_{e \tau}$ para o sistema $\varepsilon_{e \mu}-\varepsilon_{e \tau}$, (e1) $\varepsilon_{e \mu}$ e (e2) $\varepsilon_{\tau \tau}$ para o sistema $\varepsilon_{e \tau}-\varepsilon_{\tau \tau}$, e (f1) $\varepsilon_{e \tau}$ e (f2) $\varepsilon_{\tau \tau}$ para o sistema $\varepsilon_{e \tau}-\varepsilon_{\tau \tau} .111$ 
8.22 No painel superior, temos iso-contornos de $2 \sigma \mathrm{CL}$ para 2 DOF para a fase $\mathrm{CP}$, em radianos, é esperado por conseguir na fábrica de neutrinos na ausência do efeito de NSI no plano dos valores verdadeiros de $\delta$ e $\sin ^{2} 2 \theta_{13}$. A incerteza é definida por $\Delta \delta \equiv$ $\left(\delta_{\max }-\delta_{\min }\right) / 2$ (em radianos), onde $\delta_{\max }$ e $\delta_{\max }$ são os máximo e o mínimo dos valores de $\delta$ para cada ponto de input dado. O painel inferior tem um gráfico similar com o painel superior, mas com incerteza fracional $\Delta\left(\sin ^{2} 2 \theta_{13}\right) / \sin ^{2} 2 \theta_{13}$ mostrado em percentual. 112

8.23 O mesmo como no painel superior da Fig. 8.22 mais para o caso onde os parâmetros NSI de entrada não nulos. Os iso-contornos de $\Delta \delta$ (em radianos) a $2 \sigma \mathrm{CL}$ para 2DOF são mostradas para 6 combinações de sistemas com dois $\varepsilon$ : a) $\varepsilon_{e e}-\varepsilon_{e \mu}$, b) $\varepsilon_{e e}-\varepsilon_{e \tau}$, c) $\varepsilon_{e e}-\varepsilon_{\tau \tau}$, d) $\varepsilon_{e \mu}-\varepsilon_{e \tau}$ e e) $\varepsilon_{e \mu}-\varepsilon_{\tau \tau}$

$8.24 \mathrm{O}$ mesmo como no painel superior da Fig. 8.22 mas para o caso onde os parâmetros NSI de entrada não nulos. Os iso-contornos de $\Delta\left(\sin ^{2} 2 \theta_{13}\right) / \sin ^{2} 2 \theta_{13}$ a $2 \sigma$ para 2DOF são mostradas para 6 combinações de sistemas com dois $\varepsilon$ : a) $\varepsilon_{e e}-\varepsilon_{e \mu}$, b) $\varepsilon_{e e}-\varepsilon_{e \tau}$, c) $\varepsilon_{e e}-\varepsilon_{\tau \tau}$, d) $\varepsilon_{e \mu}-\varepsilon_{e \tau}$ e e) $\varepsilon_{e \mu}-\varepsilon_{\tau \tau}$. 


\section{Lista de tabelas}

3.1 Valores Característicos de $L$ e $E$ para várias fontes de neutrinos e experimentos. Adaptada de Ref. (69)

4.1 Os resultados de experimentos com neutrinos solares. Esta tabela foi obtida de (75), na qual mostra o seu déficit.

4.2 Os resultados da razão $R_{O b s / M C}$.

5.1 Os limites correntes e futuros para os parâmetros NSI separadamente nos processos de produção e detecção que mantêm sabor com $90 \%$ CL. Adaptada de Ref. (49).

5.2 Os limites correntes e futuros para os parâmetros NSI que trocam sabor com 90\% CL. Adaptada de Ref. (49). 


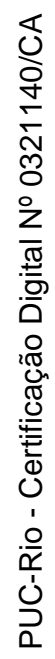

Pure logical thinking cannot yields us any knowledge of the empirical world, all knownledge of reality starts from experience and in it.

Albert Einstein, 1933. 